\title{
Systematic construction of gapped nonliquid states
}

\author{
Xiao-Gang Wen \\ Department of Physics, Massachusetts Institute of Technology, Cambridge, Massachusetts 02139, USA
}

(Received 4 April 2020; accepted 15 June 2020; published 25 August 2020)

\begin{abstract}
Using Abelian and non-Abelian topological orders in two-dimensional (2D) space and the different ways to glue them together via their gapped boundaries, we propose a systematic way to construct three-dimensional (3D) gapped states (and in other dimensions). The resulting states are called cellular topological states, which include gapped nonliquid states, as well as gapped liquid states in some special cases. Some new fracton states with fractal excitations are constructed even using $2 \mathrm{D} Z_{2}$ topological order. More general cellular topological states can be constructed by connecting 2D domain walls between different 3D topological orders. The constructed cellular topological states can be viewed as fixed-point states for a reverse renormalization of gapped nonliquid states.
\end{abstract}

DOI: 10.1103/PhysRevResearch.2.033300

\section{INTRODUCTION}

Different phases of matter are not only characterized by their symmetry breaking patterns $[1,2]$. Even systems without any symmetry can have many distinct gapped zerotemperature phases, characterized by different patterns of long-range quantum entanglement [3]. Those gapped phases include gapped liquid phases $[4,5]$ [which include phases with topological orders [6-8], symmetry enriched topological (SET) orders [9-17], and symmetry protected trivial (SPT) orders [18-20]], as well as gapped nonliquid phases [21,22], such as foliated phases $[4,23,24]$ (i.e. type-I fracton phases [25]).

So far, we have a nearly complete understanding gapped liquid phases for boson and fermion systems with and without symmetry. In $1+1 \mathrm{D}$, all gapped phases are liquid phases. They are classified by $\left(G_{H}, G_{\Psi}, \omega_{2}\right)$ [26,27], where $G_{H}$ is the symmetry group of the Hamiltonian, $G_{\Psi}$ the symmetry group of the ground state $G_{\Psi} \subset G_{H}$, and $\omega_{2} \in H^{2}\left(G_{\Psi}, \mathbb{R} / \mathbb{Z}\right)$ is a group 2-cocycle for the unbroken symmetry group $G_{\Psi}$.

In $2+1 \mathrm{D}$, we believe that all gapped phases are liquid phases. They are classified (up to $E_{8}$ invertible topological orders and for a finite unitary on-site symmetry $G_{\Psi}$ ) by $\left(G_{H}, \mathcal{R e p}\left(G_{\Psi}\right) \subset \mathcal{C} \subset \mathcal{N}\right)$ for bosonic systems and by $\left(G_{H}, \operatorname{sRep}\left(G_{\Psi}\right) \subset \mathcal{C} \subset \mathcal{M}\right)$ for fermionic systems [28-30]. Here $\mathcal{R e p}\left(G_{\Psi}\right)$ is the symmetric fusion category formed by representations of $G_{\Psi}$, and $\operatorname{s} \mathcal{R} \operatorname{ep}\left(G_{\Psi}\right)$ is the symmetric fusion category formed by $Z_{2}^{f}$-graded (i.e., fermion graded) representations of $G_{\Psi}$. Also $\mathcal{C}$ is a braided fusion category and $\mathcal{M}$ is a minimal modular extension [29,30].

In $3+1 \mathrm{D}$, some gapped phases are liquid phases while others are nonliquid phases. The $3+1 \mathrm{D}$ gapped liquid phases

Published by the American Physical Society under the terms of the Creative Commons Attribution 4.0 International license. Further distribution of this work must maintain attribution to the author(s) and the published article's title, journal citation, and DOI. without symmetry for bosonic systems (i.e., $3+1 \mathrm{D}$ bosonic topological orders) are classified by Dijkgraak-Witten theories if the pointlike excitations are all bosons, by twisted twogauge theory with gauge 2-group $\mathcal{B}\left(G, Z_{2}\right)$ if some pointlike excitations are fermions and there are no Majorana zero modes, and by a special class of fusion 2-categories if some pointlike excitations are fermions and there are Majorana zero modes at some triple-string intersections [31-33]. Comparing with classifications of $3+1 \mathrm{D}$ SPT orders for bosonic [20,34] and fermionic systems [35-40], this result suggests that all $3+1 \mathrm{D}$ gapped liquid phases (such as SET and SPT phases) for bosonic and fermionic systems with a finite unitary symmetry (including trivial symmetry, i.e., no symmetry) are classified by partially gauging the symmetry of the bosonic/fermionic SPT orders [32].

However, the classification of gapped nonliquid phases is still unclear (for a review, see Ref. [41]). In this paper, we are going to propose a very systematic construction of $3+1 \mathrm{D}$ gapped nonliquid phases for bosonic and fermionic systems with possible symmetry. We hope our systematic construction can lead to a classifying understanding of gapped nonliquid phases. Our construction is based on the above classification of gapped liquid phases and a classification of gapped boundaries of those gapped liquid phases [42].

In a simplified form of our construction, we divide the three-dimensional (3D) space into cells, where only cell surfaces can overlap. We put a $2+1 \mathrm{D}$ topological order on each patch of overlapping surfaces. The edges can be viewed as a boundary of several stacked $2+1 \mathrm{D}$ topological orders. We put a gapped boundary (a $1+1 \mathrm{D}$ anomalous topological order [42-44]) on each edge. We refer the constructed gapped states as cellular topological states, to stress the intrinsic cellular structure (such as foliation structure $[4,23,24]$ ) in those gapped states. In general, cellular topological states are gapped nonliquid states, although some special cellular topological states can be liquid states (i.e., the cellular structure disappears).

Our construction (see Fig. 1) is similar to some constructions of SPT and SET phases [45-48]. It is also similar 


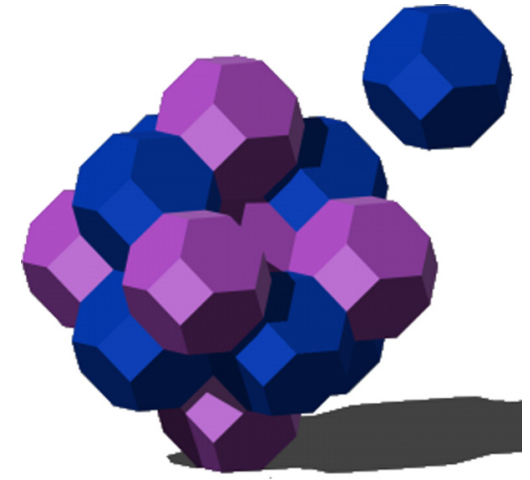

FIG. 1. The 3D space is divided into cells. Each cell surface is occupied by a $2+1 \mathrm{D}$ topological order. Each edge is occupied by an anomalous $1+1 \mathrm{D}$ topological order, which is a gapped boundary of a stacking of the $2+1 \mathrm{D}$ topological orders.

to the layer, cage-net, or string-membrane constructions of fracton phases [49-54]. However, there are some important differences, which allow us to construct new fracton states with fractal excitations [22], even starting from $2+1 \mathrm{D} Z_{2}$ topological order.

We like to mention that there are also gapless nonliquid phases. They include Fermi liquid in 2+1D and above [5], as well as some models with emergent gravitonlike excitations [55-57].

\section{A SIMPLE CONSTRUCTION}

\section{A. The construction}

We first consider a very simple decomposition of the $3 \mathrm{D}$ space into the hexagonal column's $\mathbb{R}^{3}=\cup_{i}\left(H_{i} \times \mathbb{R}_{z}\right)$, where $H_{i}$ are nonoverlapping hexagons whose union form the $x-y$ plane. (see Fig. 2). We use $i, j$ to label the vertices and $i j$ the links of the honeycomb lattice in the $x-y$ plane. We then, put bosonic topological orders $\mathcal{M}_{i j}$ without symmetry on the faces of hexagonal column's $\langle i j\rangle \times \mathbb{R}_{z}$.

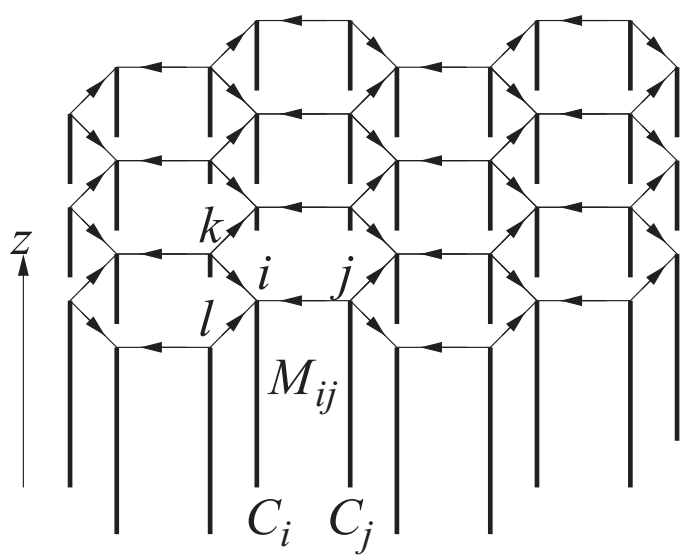

FIG. 2. The 3D space is decomposed into the hexagonal column's. The honeycomb lattice has two kinds of vertices: Type-A vertices have arrows pointing in, and type-B vertices have arrows pointing out.
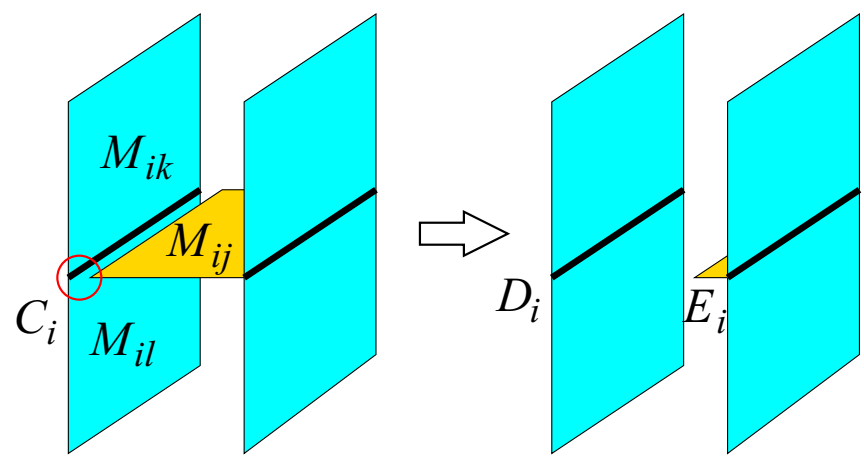

FIG. 3. For $\mathcal{C}_{i}$ satisfying Eq. (2), the layer $\mathcal{M}_{i j}$ is detached.

We note that the vertical line at the vertex $i$ of the honeycomb lattice is the boundary of the topological order $\mathcal{M}_{i j} \otimes$ $\mathcal{M}_{i k} \otimes \mathcal{M}_{i l}$. (Here $\mathcal{C} \otimes \mathcal{D}$ is the topological order obtained by stacking topological orders $\mathcal{C}$ and $\mathcal{D}$.) So in general, we can put a $1+1 \mathrm{D}$ anomalous topological order [42-44] on the vertical line $i$ which is described by a fusion category $\mathcal{C}_{i}$ [58]. Those fusion categories satisfy

$$
\begin{aligned}
\mathcal{Z}\left(\mathcal{C}_{i}\right) & = \begin{cases}\mathcal{M}_{i}, & \text { if } i \text { is type } \mathrm{A}, \\
\mathcal{M}_{i}, & \text { if } i \text { is type } \mathrm{B},\end{cases} \\
\mathcal{M}_{i} & =\mathcal{M}_{i j} \otimes \mathcal{M}_{i k} \otimes \mathcal{M}_{i l},
\end{aligned}
$$

which means that $1+1 \mathrm{D}$ anomalous topological order $\mathcal{C}_{i}$ is a gapped boundary of $2+1 \mathrm{D}$ topological order $\mathcal{M}_{i}$ or $\overline{\mathcal{M}}_{i}$ [42,59-61]. Here $\mathcal{Z}$ is the Drinfeld center. A physical calculation of the Drinfeld center is presented in Ref. [58]. Also, the bar means the time reversal conjugate.

We see that, using the data $\left(\mathcal{M}_{i j}, \mathcal{C}_{i}\right)$, we can construct a $3+1 \mathrm{D}$ gapped phase for bosonic systems, which can be a nonliquid gapped phase. In general $\mathcal{M}_{i j}$ can be non-Abelian topological orders.

We like to mention that if, for example, $\mathcal{C}_{i}$ has a form,

$$
\begin{aligned}
\mathcal{C}_{i} & =\mathcal{D}_{i} \otimes \mathcal{E}_{i}, \\
\mathcal{Z}\left(\mathcal{E}_{i}\right) & =\mathcal{M}_{i j}, \\
\mathcal{Z}\left(\mathcal{D}_{i}\right) & =\mathcal{M}_{i k} \otimes \mathcal{M}_{i l},
\end{aligned}
$$

then the layer $\mathcal{M}_{i j}$ is not connected to the line at vertex $i$. Such a layer can shrink to the line at vertex $j$ on the other side (see Fig. 3), and hence can be removed (or correspond to the trivial $\mathcal{M}_{i j}$ case). Thus we are looking for the so-called entangled solutions of Eq. (1) that do not have the form (2). We also like to mention that the gapped boundaries of a $2+1 \mathrm{D}$ topological order can be constructed via anyon condensation and are classified by the Lagrangian algebra of the $2+1 \mathrm{D}$ topological order [62-67].

\section{B. Entanglement structure}

To understand the cellular topological state in Fig. 2 better, we like to study the entanglement structure of the cellular topological state. The entanglement structure can be revealed by the renormalization of the state (see Fig. 4). The renormalization is done via a basic deformation step in Fig. 4(a), where fusing two boundaries $\mathcal{C}, \mathcal{D}$ and fusing two boundaries $\mathcal{C}^{\prime}, \mathcal{D}^{\prime}$ given rise to the same boundary of the four stacked 


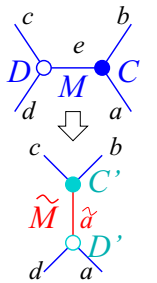

(a)

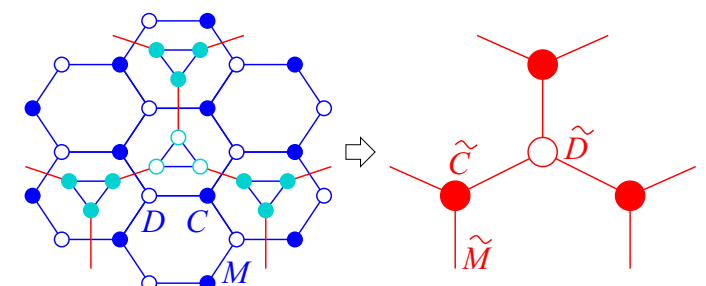

(b) (c)
FIG. 4. (a) A deformation step $\mathcal{C} \otimes_{\mathcal{M}} \mathcal{D}=\mathcal{C}^{\prime} \otimes_{\tilde{\mathcal{M}}} \mathcal{D}^{\prime} \quad$ [see Eq. (11)]. (b) Using the deformation step, we can change the bluehexagonal tensor network to the one formed by red links and lightblue dots. (c) Shrinking the triangles to the red dots produces the blue-hexagonal tensor network [see Eq. (12)]. This completes a renormalization step $(\mathcal{M}, \mathcal{C}, \mathcal{D}) \rightarrow(\tilde{\mathcal{M}}, \tilde{\mathcal{C}}, \tilde{\mathcal{D}})$.

$2+1 \mathrm{D}$ topological orders (described by the four outer lines): $\mathcal{C} \otimes_{\mathcal{M}} \mathcal{D}=\mathcal{C}^{\prime} \otimes_{\tilde{\mathcal{M}}} \mathcal{D}^{\prime}$

To describe such a deformation step more explicitly, we need a quantitative description of the $2+1 \mathrm{D}$ topological order $\mathcal{M}_{i j}$ and the $1+1 \mathrm{D}$ anomalous topological order $\mathcal{C}_{i}$. The topological orders can be characterized by the representations of mapping class groups for all Riemannian surfaces [7,8]. Here, for simplicity [68-70], we will only use the representation for mapping the class group of a torus [71,72]. In other words, we will use the $S_{a}^{b}, T_{a}^{b}$ matrices [the generators of a modular representation of $S L(2, \mathbb{Z})$ ] to characterize a $2+1 \mathrm{D}$ topological order $\mathcal{M}$, where $a, b$ label the types of the topological excitations in the topological order. Similarly, the gapped domain walls $\mathcal{C}$ between two topological orders characterized by $(S, T)$ and $\left(S^{\prime}, T^{\prime}\right)$ are characterized by the wave function overlap of the degenerate ground states, $\left|\psi_{a}\right\rangle$ and $\left|\psi_{a^{\prime}}^{\prime}\right\rangle$, of the two topological orders on torus [61]:

$$
\left\langle\psi_{a^{\prime}}^{\prime}\left|e^{-H_{W}}\right| \psi_{a}\right\rangle=e^{-\sigma A_{T^{2}}+o\left(\frac{1}{A_{T^{2}}}\right)} C_{a^{\prime}}^{a},
$$

where $H_{W}$ is the local Hermitian operator like a Hamiltonian of a quantum system, $A_{T^{2}}$ is the area of the torus $T^{2}$, and $C_{a^{\prime}}^{a}$ is a topological invariant that characterizes the domain between the two topological orders. $C_{a^{\prime}}^{a}$ turns out to be non-negative integers for torus, which satisfy $[59,61]$

$$
\begin{aligned}
\sum_{b^{\prime}}{S^{\prime}}_{a^{\prime}}^{b^{\prime}} C_{b^{\prime}}^{b} & =\sum_{a} C_{a^{\prime}}^{a} S_{a}^{b}, \\
\sum_{b^{\prime}}{T^{\prime}}_{a^{\prime}}^{b^{\prime}} C_{b^{\prime}}^{b} & =\sum_{a} C_{a^{\prime}}^{a} T_{a}^{b}, \\
C_{a^{\prime}}^{a} C_{b^{\prime}}^{b} & \leqslant \sum_{c^{\prime}, c} N_{c^{\prime}}^{a^{\prime} b^{\prime}} C_{c^{\prime}}^{c} N_{c}^{a b} .
\end{aligned}
$$

where $N_{c}^{a b}$ and $N_{c^{\prime}}^{a^{\prime} b^{\prime}}$ are the fusion coefficients for the topological excitations in the two topological orders.

Now let us describe an elementary deformation step. Consider three topological orders $\mathcal{M}, \mathcal{M}^{\prime}$, and $\mathcal{M}^{\prime \prime}$ characterized by $(S, T),\left(S^{\prime}, T^{\prime}\right)$, and $\left(S^{\prime \prime}, T^{\prime \prime}\right)$. A tensor $C$ describes a domain wall $\mathcal{C}$ between $(S, T)$ and $\left(S^{\prime}, T^{\prime}\right)$, and a tensor $C^{\prime}$ describes a domain wall $\mathcal{C}^{\prime}$ between $\left(S^{\prime}, T^{\prime}\right)$ and $\left(S^{\prime \prime}, T^{\prime \prime}\right)$. The two domain walls $\mathcal{C}$ and $\mathcal{C}^{\prime}$ can fuse into a single domain wall $\mathcal{C}^{\prime \prime}$ [see Fig. 5(a)]:

$$
\mathcal{C}^{\prime \prime}=\mathcal{C}^{\prime} \otimes_{\mathcal{M}^{\prime}} \mathcal{C}
$$

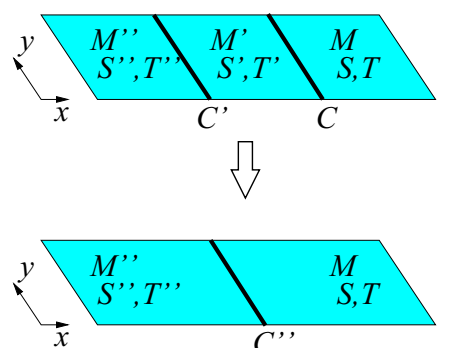

(a)

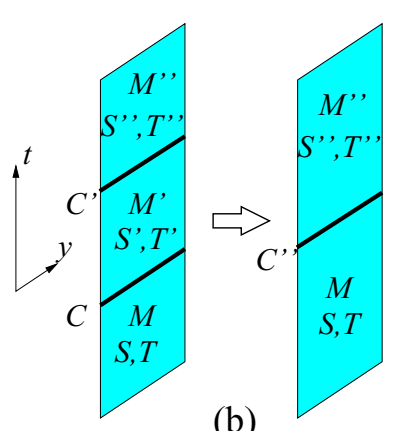

(b)
FIG. 5. (a) Fusion of two $1+1 \mathrm{D}$ domain walls $\mathcal{C}$ and $\mathcal{C}^{\prime}$ connected by a $2+1 \mathrm{D}$ topological order $\mathcal{M}^{\prime}$ gives rise to $\mathcal{C}^{\prime \prime}=\mathcal{C}^{\prime} \otimes_{\mathcal{M}^{\prime}}$ $\mathcal{C}$. The looplike $t$ direction is not shown. (b) Exchanging $x$ and $t$, we get the corresponding wave function overlaps. Two wave-function overlaps $C$ and $C^{\prime}$ can be reduced to one wave-function overlap $C^{\prime \prime}$. The looplike $x$ direction is not shown.

Note the $\mathcal{C}$ and $\mathcal{C}^{\prime}$ are fused with a "glue" $\mathcal{M}{ }^{\prime}$ (see Fig. 5) $[73,74]$, which is indicated by the subscript of $\otimes$. It turns out that the domain wall $\mathcal{C}^{\prime \prime}$ is characterized by a tensor $C^{\prime \prime}$,

$$
\left(C^{\prime \prime}\right)_{a^{\prime \prime}}^{a}=\sum_{a^{\prime}}\left(C^{\prime}\right)_{a^{\prime \prime}}^{a^{\prime}} C_{a^{\prime}}^{a} \text {, or } C^{\prime \prime}=C^{\prime} C .
$$

The above just describes the composition of wave-function overlap in Fig. 5(b).

We note that the above elementary step is reversible, which can fuse two domain walls or split a single domain wall. A fusion following a split in a different direction produces the elementary deformation step in Fig. 4(a).

If one side of the domain wall between $\mathcal{M}$ and $\mathcal{M}{ }^{\prime}$ is trivial (say $\mathcal{M}^{\prime}$ is trivial), then the domain wall (i.e., the boundary of $\mathcal{M}$ ) is described by $C_{1}^{a} \equiv C^{a}$ (or by $C_{a}^{1} \equiv C_{a}$ if the boundary is at the opposite side of $\mathcal{M}$, where $\mathbf{1}$ corresponds to the trivial excitation). We see that the boundary $\mathcal{C}_{i}$ in our construction (see Fig. 2) is characterized by non-negative integer tensor,

$$
\mathcal{C}_{i} \sim \begin{cases}C_{a_{i j} a_{i k} a_{i l}}, & \text { if } i \text { is type A, } \\ C^{a_{i j} a_{i k} a_{i l}}, & \text { if } i \text { is type B, }\end{cases}
$$

where $\left(a_{i j}, a_{i k}, a_{i l}\right)$ labels the topological excitations in $\mathcal{M}_{i}=$ $\mathcal{M}_{i j} \otimes \mathcal{M}_{i k} \otimes \mathcal{M}_{i l}$ and $a_{i j}$ labels the topological excitations in $\mathcal{M}_{i j}$, etc.

The above discussion suggests that we can view the blue honeycomb lattice in Fig. 4 as a tensor network, where the tensors at the solid blue vertices are given by $C^{a_{i j} a_{i k} a_{i l}}$, while the tensors at the open blue vertices are given by $D_{a_{i j} a_{i k} a_{i l}}$. The link $\langle i j\rangle$ carries the index $a_{i j}$ which labels the types of topological excitations in $\mathcal{M}_{i j}$. The trace of the tensor network gives us the partition function, which is the ground-state degeneracy of the cellular topological state $[59,61,64]$.

To see why the trace of the tensor network gives rise to ground-state degeneracy, let us consider a simple tensor network with two vertices connected by a link $[59,64]$. The link corresponds to a $Z_{2}$ topological order $\mathcal{M}=\mathcal{G}_{Z_{2}}^{2+1}$ (i.e., the $2+1 \mathrm{D} Z_{2}$ gauge theory) $[75,76]$. The $Z_{2}$ topological order $\mathcal{G}_{Z_{2}}^{2+1}$ has four types of topological excitations $\mathbf{1}, e, m, f$, labeled by $a=1,2,3,4$, respectively. The $S, T$ modular 


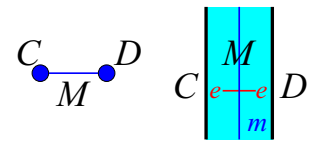

FIG. 6. A simple tensor network formed by two vertices connected by a link. The link corresponds to a $2+1 \mathrm{D}$ topological order $\mathcal{M}$. The vertices corresponds to a $1+1 \mathrm{D}$ anomalous topological order $\mathcal{C}, \mathcal{D}$.

matrices are given by

$$
T=\left(\begin{array}{cccc}
1 & 0 & 0 & 0 \\
0 & 1 & 0 & 0 \\
0 & 0 & 1 & 0 \\
0 & 0 & 0 & -1
\end{array}\right), \quad S=\frac{1}{2}\left(\begin{array}{rrrr}
1 & 1 & 1 & 1 \\
1 & 1 & -1 & -1 \\
1 & -1 & 1 & -1 \\
1 & -1 & -1 & 1
\end{array}\right)
$$

The $Z_{2}$ topological order has two gapped boundaries: $\mathcal{E}$ from $e$-particle condensation and $\mathcal{M}$ from $m$-particle condensation [63]. There are described by the following rank-1 tensors:

$$
\mathcal{E}:\left(E^{a}\right)=\left(\begin{array}{l}
1 \\
1 \\
0 \\
0
\end{array}\right), \quad \mathcal{M}:\left(M^{a}\right)=\left(\begin{array}{l}
1 \\
0 \\
1 \\
0
\end{array}\right) .
$$

If both boundaries in Fig. 6 are given by $\mathcal{C}=\mathcal{D}=\mathcal{E}$, then the ground-state degeneracy of the system is given by $\sum_{a} E^{a} E^{a}=2$. This result can also be obtained using the $e$-string operator $W_{e}$ that creates a pair of $e$ particles at its ends, and the $m$-string operator $W_{m}$ that creates a pairs of $m$ particles at its ends. Since $e$ particles condense at the boundaries, the open $e$-string operator $W_{e}$ connecting the two boundaries does change the energy (i.e., commute with the Hamiltonian). A loop of the $m$-string operator in the $z$ direction $W_{m}^{z}$ also commutes with the Hamiltonian. Since the $e$-string operator and the $m$-string operator intersect at one point and anticommute $W_{e} W_{m}^{z}=-W_{m}^{z} W_{e}$, the ground states are twofold degenerate.

If the boundaries in Fig. 6 are given by $\mathcal{C}=\mathcal{E}$ and $\mathcal{C}=$ $\mathcal{M}$, then the ground-state degeneracy of the system is given by $\sum_{a} E^{a} M^{a}=1$. In this case, there is no string operator that connects the two boundaries and create two condensing particles.

With the above tensor representation of the boundaries, the renormalization of the cellular topological state becomes the standard renormalization of tensor network [77,78]. Let us assume that, in the hexagonal tensor network [see Figs. 2 and 4(b)], all $\mathcal{M}_{i j}$ are the same $\mathcal{M}_{i j}=\mathcal{M}$, whose topological excitations are labeled by $a, b, c, \ldots$ The boundaries $\mathcal{C}_{i}$ at the solid blue vertices are given by $\mathcal{C}_{i}=\mathcal{C}$ (or by tensor $C^{a b c}$ ), while boundaries $\mathcal{C}_{i}$ at the open blue vertices are given by $\mathcal{C}_{i}=\mathcal{D}$ (or by tensor $D_{a b c}$ ). For simplicity, we will assume

$$
C^{a b c}=C^{c a b}, \quad D_{a b c}=D_{c a b} .
$$

Then, the deformation in Fig. 4(a) is explicitly given by the following tensor relation:

$$
\sum_{e} C^{e a b} D_{e c d}=\sum_{\tilde{a}}\left(C^{\prime}\right)^{\tilde{a} b c}\left(D^{\prime}\right)_{\tilde{a} d a},
$$

where $\tilde{a}$ labels the topological excitations in a new $2+1 \mathrm{D}$ topological order $\tilde{\mathcal{M}}$. We like to mention that the deformation
(11) is not unique. There can be many choices of $\tilde{\mathcal{M}}, \mathcal{C}^{\prime}, \mathcal{D}^{\prime}$ that satisfy Eq. (11). We like to find the deformation where $\tilde{\mathcal{M}}$ has minimal total quantum dimension $D=\sqrt{\sum_{\tilde{a}} d_{\tilde{a}}^{2}}$. Here $d_{\tilde{a}}$ is the quantum dimensions of topological excitations in $\tilde{\mathcal{M}}$. Later, we will see that if the resulting $\tilde{\mathcal{M}}$ is trivial or equal to the original $\mathcal{M}$, then the corresponding cellular topological state may be a liquid state.

We like to remark that, as we will see later, a cellular state contains extra local structures that are not related to the universal class of a gapped state. So, by choosing $\tilde{\mathcal{M}}$ to have minimal total quantum dimension, we hope to obtain the simplest cellular topological state after each step of renormalization, trying to remove those local structures as much as possible.

We can use the deformation Fig. 4(a) to deform the blue hexagonal tensor network in Fig. 4(b) to the one described by red links in Fig. 4(b). We then shrink the small triangles in Fig. 4(b) to a point and obtain a new red hexagonal tensor network in Fig. 4(c). The new boundaries $\tilde{\mathcal{C}}$ and $\tilde{\mathcal{D}}$ are given by

$$
\begin{aligned}
\tilde{C}^{\tilde{a} \tilde{b} \tilde{c}} & =\sum_{a, b, c}\left(C^{\prime}\right)^{\tilde{a} c b}\left(C^{\prime}\right)^{\tilde{b} a c}\left(C^{\prime}\right)^{\tilde{c} b a}, \\
\tilde{D}_{\tilde{a} \tilde{b} \tilde{c}} & =\sum_{a, b, c}\left(D^{\prime}\right)_{\tilde{a} c b}\left(D^{\prime}\right)_{\tilde{b} a c}\left(D^{\prime}\right)_{\tilde{c} b a} .
\end{aligned}
$$

The two relations (11) and (12) define the renormalization of the cellular topological state.

\section{CELLULAR TOPOLOGICAL STATES FROM $2+1 D Z_{2}$ TOPOLOGICAL ORDER}

\section{A. A general construction}

In this section, we are going to construct some simple cellular topological states in Fig. 2 by choosing $\mathcal{M}_{i j}$ to be the same $2+1 \mathrm{D} Z_{2}$ topological order $\mathcal{M}_{i j}=\mathcal{G}_{Z_{2}}^{2+1}$. We find that $\mathcal{G}_{Z_{2}}^{2+1} \otimes \mathcal{G}_{Z_{2}}^{2+1} \otimes \mathcal{G}_{Z_{2}}^{2+1}$ has 10 types of gapped boundaries, $\mathcal{Z}\left(\mathcal{C}_{i}\right)=\mathcal{G}_{Z_{2}}^{2+1} \otimes \mathcal{G}_{Z_{2}}^{2+1} \otimes \mathcal{G}_{Z_{2}}^{2+1}$, that are entangled [i.e., do not have the form in Eq. (2)]. Their tensor representations $C_{i}^{a b c}$ are given by (only nonzero elements are listed)

$$
\begin{aligned}
& \mathcal{C}_{1}: C_{1}^{111}, C_{1}^{122}, C_{1}^{212}, C_{1}^{221}, C_{1}^{333}, C_{1}^{344}, C_{1}^{434}, C_{1}^{443}=1, \\
& \mathcal{C}_{2}: C_{2}^{111}, C_{2}^{144}, C_{2}^{223}, C_{2}^{232}, C_{2}^{322}, C_{2}^{333}, C_{2}^{414}, C_{2}^{441}=1, \\
& \mathcal{C}_{3}: C_{3}^{111}, C_{3}^{133}, C_{3}^{222}, C_{3}^{244}, C_{3}^{313}, C_{3}^{331}, C_{3}^{424}, C_{3}^{442}=1, \\
& \mathcal{C}_{4}: C_{4}^{111}, C_{4}^{144}, C_{4}^{222}, C_{4}^{233}, C_{4}^{323}, C_{4}^{332}, C_{4}^{414}, C_{4}^{441}=1, \\
& \mathcal{C}_{5}: C_{5}^{111}, C_{5}^{132}, C_{5}^{212}, C_{5}^{231}, C_{5}^{323}, C_{5}^{344}, C_{5}^{424}, C_{5}^{443}=1, \\
& \mathcal{C}_{6}: C_{6}^{111}, C_{6}^{132}, C_{6}^{223}, C_{6}^{244}, C_{6}^{312}, C_{6}^{331}, C_{6}^{424}, C_{6}^{443}=1, \\
& \mathcal{C}_{7}: C_{7}^{111}, C_{7}^{123}, C_{7}^{213}, C_{7}^{221}, C_{7}^{332}, C_{7}^{344}, C_{7}^{434}, C_{7}^{442}=1, \\
& \mathcal{C}_{8}: C_{8}^{111}, C_{8}^{123}, C_{8}^{232}, C_{8}^{244}, C_{8}^{313}, C_{8}^{321}, C_{8}^{434}, C_{8}^{442}=1, \\
& \mathcal{C}_{9}: C_{9}^{111}, C_{9}^{133}, C_{9}^{213}, C_{9}^{231}, C_{9}^{322}, C_{9}^{344}, C_{9}^{424}, C_{9}^{442}=1, \\
& \mathcal{C}_{10}: C_{10}^{111}, C_{10}^{122}, C_{10}^{233}, C_{10}^{244}, C_{10}^{312}, C_{10}^{321}, C_{10}^{434}, C_{10}^{443}=1 .
\end{aligned}
$$

The first four, $\left(\mathcal{C}_{1}, \mathcal{C}_{2}, \mathcal{C}_{3}, \mathcal{C}_{4}\right)$, are cyclic symmetric (10). Let us examine those four types of the boundaries of 


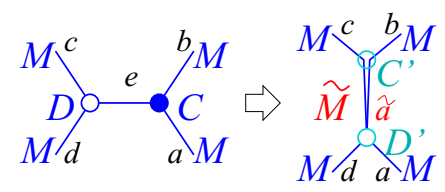

FIG. 7. If we choose $\tilde{\mathcal{M}}=\mathcal{M} \otimes \mathcal{M}$, then Eq. (11) always has solutions.

$\mathcal{G}_{Z_{2}}^{2+1} \otimes \mathcal{G}_{Z_{2}}^{2+1} \otimes \mathcal{G}_{Z_{2}}^{2+1}$ in more detail. First we note that $\mathcal{C}_{1}$ and $\mathcal{C}_{3}$ as well as $\mathcal{C}_{2}$ and $\mathcal{C}_{4}$ differ by an automorphism of the $Z_{2}$ topological order: $e_{i} \leftrightarrow m_{i}$, where $i=1,2,3$ labels the three $Z_{2}$ topological orders in $\mathcal{G}_{Z_{2}}^{2+1} \otimes \mathcal{G}_{Z_{2}}^{2+1} \otimes \mathcal{G}_{Z_{2}}^{2+1}$. Those boundaries are formed by condensing the topological excitations in the three $Z_{2}$ topological orders [63,65-67]. In the following, we list the condensing excitations (the generators) for the four boundaries:

$$
\begin{aligned}
& \mathcal{C}_{1}: e_{2} e_{3}, \quad e_{1} e_{3}, \quad e_{1} e_{2}, \quad m_{1} m_{2} m_{3} ; \\
& \mathcal{C}_{2}: f_{1} f_{2}, \quad f_{1} f_{3}, \quad f_{2} f_{3}, \quad m_{1} m_{2} m_{3} \text {; } \\
& \mathcal{C}_{3}: m_{2} m_{3}, \quad m_{1} m_{3}, \quad m_{1} m_{2}, \quad e_{1} e_{2} e_{3} \text {; } \\
& \mathcal{C}_{4}: f_{2} f_{3}, \quad f_{1} f_{3}, \quad f_{1} f_{2}, \quad e_{1} e_{2} e_{3} \text {; }
\end{aligned}
$$

which are obtained from the tensor indices $a b c$ with $C^{a b c}=1$.

If we assume $\tilde{\mathcal{M}}$ to be the trival topological order, then Eq. (11) for the deformation in Fig. 11(a) has no solutions for those cyclic symmetric boundaries. If we assume $\tilde{\mathcal{M}}$ to be given by the $2+1 \mathrm{D} Z_{2}$ topological order $\mathcal{G}_{Z_{2}}^{2+1}$, then for the following $(\mathcal{C}, \mathcal{D})$ 's:

$$
\left(\mathcal{C}_{1}, \mathcal{C}_{2}\right),\left(\mathcal{C}_{1}, \mathcal{C}_{3}\right),\left(\mathcal{C}_{1}, \mathcal{C}_{4}\right),\left(\mathcal{C}_{2}, \mathcal{C}_{3}\right),\left(\mathcal{C}_{3}, \mathcal{C}_{4}\right),
$$

the deformation Eq. (11) also has no solution. But, for other cyclic symmetric boundaries $\mathcal{C}, \mathcal{D}$ 's, the deformation Eq. (11) has two solutions, which are given by (see Fig. 11)

$$
\begin{aligned}
& (\mathcal{C}, \mathcal{D})=\left(\mathcal{C}_{1}, \mathcal{C}_{1}\right) \rightarrow\left(\mathcal{C}^{\prime}, \mathcal{D}^{\prime}\right)=\left(\mathcal{C}_{1}, \mathcal{C}_{1}\right) \text { or }\left(\mathcal{C}_{10}, \mathcal{C}_{10}\right), \\
& (\mathcal{C}, \mathcal{D})=\left(\mathcal{C}_{3}, \mathcal{C}_{3}\right) \rightarrow\left(\mathcal{C}^{\prime}, \mathcal{D}^{\prime}\right)=\left(\mathcal{C}_{3}, \mathcal{C}_{3}\right) \text { or }\left(\mathcal{C}_{9}, \mathcal{C}_{9}\right), \\
& (\mathcal{C}, \mathcal{D})=\left(\mathcal{C}_{2}, \mathcal{C}_{2}\right) \rightarrow\left(\mathcal{C}^{\prime}, \mathcal{D}^{\prime}\right)=\left(\mathcal{C}_{2}, \mathcal{C}_{2}\right) \text { or }\left(\mathcal{C}_{4}, \mathcal{C}_{4}\right), \\
& (\mathcal{C}, \mathcal{D})=\left(\mathcal{C}_{4}, \mathcal{C}_{4}\right) \rightarrow\left(\mathcal{C}^{\prime}, \mathcal{D}^{\prime}\right)=\left(\mathcal{C}_{2}, \mathcal{C}_{2}\right) \text { or }\left(\mathcal{C}_{4}, \mathcal{C}_{4}\right), \\
& (\mathcal{C}, \mathcal{D})=\left(\mathcal{C}_{2}, \mathcal{C}_{4}\right) \rightarrow\left(\mathcal{C}^{\prime}, \mathcal{D}^{\prime}\right)=\left(\mathcal{C}_{2}, \mathcal{C}_{4}\right) \text { or }\left(\mathcal{C}_{4}, \mathcal{C}_{2}\right)
\end{aligned}
$$

If we choose $\tilde{\mathcal{M}}$ to be a more general topological order, such as $\tilde{\mathcal{M}}=\mathcal{M} \otimes \mathcal{M}$, then Eq. (11) always has solutions (see Fig. 7).

After obtaining $\mathcal{C}^{\prime}$ and $\mathcal{D}^{\prime}$, we can perform the shrinking operation (12) (see Fig. 4 ) to obtain $\tilde{\mathcal{C}}, \tilde{\mathcal{D}}$ :

$$
\begin{aligned}
& (\mathcal{C}, \mathcal{D})=\left(\mathcal{C}_{1}, \mathcal{C}_{1}\right) \rightarrow(\tilde{\mathcal{C}}, \tilde{\mathcal{D}})=\left(2 \mathcal{C}_{1}, 2 \mathcal{C}_{1}\right) \text { or }\left(2 \mathcal{C}_{3}, 2 \mathcal{C}_{3}\right), \\
& (\mathcal{C}, \mathcal{D})=\left(\mathcal{C}_{3}, \mathcal{C}_{3}\right) \rightarrow(\tilde{\mathcal{C}}, \tilde{\mathcal{D}})=\left(2 \mathcal{C}_{3}, 2 \mathcal{C}_{3}\right) \text { or }\left(2 \mathcal{C}_{1}, 2 \mathcal{C}_{1}\right), \\
& (\mathcal{C}, \mathcal{D})=\left(\mathcal{C}_{2}, \mathcal{C}_{2}\right) \rightarrow(\tilde{\mathcal{C}}, \tilde{\mathcal{D}})=\left(2 \mathcal{C}_{2}, 2 \mathcal{C}_{2}\right) \text { or }\left(2 \mathcal{C}_{4}, 2 \mathcal{C}_{4}\right), \\
& (\mathcal{C}, \mathcal{D})=\left(\mathcal{C}_{4}, \mathcal{C}_{4}\right) \rightarrow(\tilde{\mathcal{C}}, \tilde{\mathcal{D}})=\left(2 \mathcal{C}_{2}, 2 \mathcal{C}_{2}\right) \text { or }\left(2 \mathcal{C}_{4}, 2 \mathcal{C}_{4}\right), \\
& (\mathcal{C}, \mathcal{D})=\left(\mathcal{C}_{2}, \mathcal{C}_{4}\right) \rightarrow(\tilde{\mathcal{C}}, \tilde{\mathcal{D}})=\left(2 \mathcal{C}_{2}, 2 \mathcal{C}_{4}\right) \text { or }\left(2 \mathcal{C}_{4}, 2 \mathcal{C}_{2}\right) .
\end{aligned}
$$

Here $2 \mathcal{C} \equiv \mathcal{C} \oplus \mathcal{C}$ means that the boundary is formed by accidentally degenerate $\mathcal{C}$ and $\mathcal{C}$, since $\tilde{\mathcal{C}}$ comes from fusing three $\mathcal{C}$ 's. We roughly have a fusion rule for the boundaries: $\mathcal{C} \otimes \mathcal{C} \otimes \mathcal{C} \sim \tilde{\mathcal{C}}$. The results (17) suggest that the boundary $\mathcal{C}$ and $\mathcal{D}$ have a quantum dimension $\sqrt{2}$. So the ground-state degeneracy is roughly given by $2^{\frac{N_{A}+N_{B}}{2}}$ (up to a finite factor), where $N_{A}$ and $N_{B}$ are the number of type-A and type-B vertices (see Fig. 2). In other words the ground-state degeneracy is roughly given by $2^{N_{h}}$ where $N_{h}$ is the number of the hexagons (see Fig. 2).

In our above discussions, we have assumed that the vertices in the honeycomb lattice (see Fig. 2) is far apart. This leads to the accidental degeneracy of two $\mathcal{C}$ 's. However, in reality, the vertices in the honeycomb lattice have a small separation. In this case, the degeneracy of two $\mathcal{C}$ 's is split.

To summarize, we constructed five cellular topological phases labeled by the following $(\mathcal{M}, \mathcal{C}, \mathcal{D})$ 's:

$$
\begin{array}{ll}
\left(\mathcal{G}_{Z_{2}}^{2+1}, \mathcal{C}_{1}, \mathcal{C}_{1}\right), & \left(\mathcal{G}_{Z_{2}}^{2+1}, \mathcal{C}_{2}, \mathcal{C}_{2}\right), \\
\left(\mathcal{G}_{Z_{2}}^{2+1}, \mathcal{C}_{4}, \mathcal{C}_{4}\right), & \left(\mathcal{G}_{Z_{2}}^{2+1}, \mathcal{C}_{3}, \mathcal{C}_{3}\right),
\end{array}
$$

Those phases have the key properties that under the renormalization $(\mathcal{M}, \mathcal{C}, \mathcal{D}) \rightarrow(\tilde{\mathcal{M}}, \tilde{\mathcal{C}}, \tilde{\mathcal{D}})$ in Fig. 4, we cannot reduce the $2+1 \mathrm{D}$ topological order $\mathcal{M}$ to the trivial one, but $\mathcal{M}$ can be unchanged under renormalization: $\mathcal{M}=\mathcal{G}_{Z_{2}}^{2+1} \rightarrow \tilde{\mathcal{M}}=$ $\mathcal{G}_{Z_{2}}^{2+1}$. Later, we will see that the invariance of $\mathcal{M}$ under renormalization suggests that the corresponding cellular topological state is a liquid state.

We also constructed five cellular topological phases labeled by the following $(\mathcal{M}, \mathcal{C}, \mathcal{D})$ 's:

$$
\begin{array}{ll}
\left(\mathcal{G}_{Z_{2}}^{2+1}, \mathcal{C}_{1}, \mathcal{C}_{2}\right), & \left(\mathcal{G}_{Z_{2}}^{2+1}, \mathcal{C}_{1}, \mathcal{C}_{3}\right), \\
\left(\mathcal{G}_{Z_{2}}^{2+1}, \mathcal{C}_{2}, \mathcal{C}_{3}\right), & \left(\mathcal{G}_{Z_{2}}^{2+1}, \mathcal{C}_{3}, \mathcal{C}_{4}\right)
\end{array}
$$

Those phases have the key properties that under the renormalization $(\mathcal{M}, \mathcal{C}, \mathcal{D}) \rightarrow(\tilde{\mathcal{M}}, \tilde{\mathcal{C}}, \tilde{\mathcal{D}})$ in Fig. 4 , we cannot reduce the $2+1 \mathrm{D}$ topological order $\mathcal{M}$ to the trivial one, and $\mathcal{M}$ cannot be unchanged under the renormalization. Later, we will see that the noninvariance of $\mathcal{M}$ under renormalization suggests that the corresponding cellular topological state is a nonliquid state.

\section{B. Cellular topological state $\left(\mathcal{G}_{Z_{2}}^{2+1}, \mathcal{C}_{1}, \mathcal{C}_{1}\right)$}

Some cellular topological states are nonliquid states, while other cellular topological states are actually liquid states. In this section, we are going to discuss a cellular topological state $\left(\mathcal{G}_{Z_{2}}^{2+1}, \mathcal{C}_{1}, \mathcal{C}_{1}\right)$, and show that it is actually a gapped liquid state-a $3+1 \mathrm{D} Z_{2}$ topological ordered state $\mathcal{G}_{Z_{2}}^{3+1}$ described by $Z_{2}$ gauge theory.

The cellular topological state $\left(\mathcal{G}_{Z_{2}}^{2+1}, \mathcal{C}_{1}, \mathcal{C}_{1}\right)$ is constructed using $2+1 \mathrm{D} Z_{2}$ topological order, and choosing the junction of three $Z_{2}$ topological orders to be the $1+1 \mathrm{D}$ anomalous topological order $\mathcal{C}_{1}$ in Eq. (13) (see Fig. 2).

The $1+1 \mathrm{D}$ topological order $\mathcal{C}_{1}$ has a condensation of $e_{1} e_{2}, e_{2} e_{3}$, and $e_{3} e_{1}$, for the excisions in the connected $2+1 \mathrm{D}$ topological order. This means that the $e$ particles can freely move between the $2+1 \mathrm{D}$ topological orders $\mathcal{G}_{Z_{2}}^{2+1}$ connected by the $1+1 \mathrm{D}$ topological order $\mathcal{C}_{1}$ (see Fig. 8). In other words, the $e$ particle can move freely in the whole 3D space. 


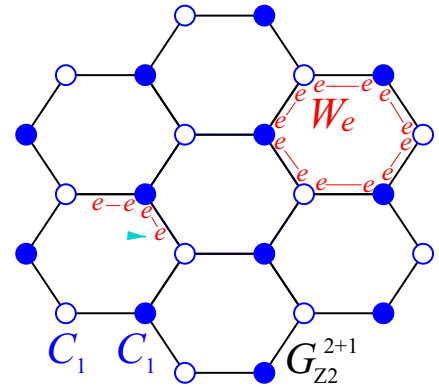

FIG. 8. In the cellular topological state $\left(\mathcal{G}_{Z_{2}}^{2+1}, \mathcal{C}_{1}, \mathcal{C}_{1}\right)$, the $e$ particle can move freely in 3D space. The unmarked links are in sector 1. A configuration with a loop of links in sector 2 (marked by $e-e$ ) corresponds to another degenerate ground state.

From the renormalization of the corresponding tensor network,

$$
(\mathcal{C}, \mathcal{D})=\left(\mathcal{C}_{1}, \mathcal{C}_{1}\right) \rightarrow(\tilde{\mathcal{C}}, \tilde{\mathcal{D}})=\left(2 \mathcal{C}_{1}, 2 \mathcal{C}_{1}\right)
$$

we find that the ground-state degeneracy is roughly given by $2^{N_{h}}$. Such degeneracy can be understood by using the closed $e$-string operator $W_{e}$ that moves an $e$ particle around a hexagon and the closed $m$-string operators $W_{m}^{z}$ that wrap around in the $z$ direction (see Fig. 6). Both closed string operators commute with the Hamiltonian. Since $W_{e}$ and $W_{m}^{z}$ anticommute when they intersect, we find that each hexagon contributes a factor 2 (corresponding to $W_{e}= \pm 1$ ) to the ground-state degeneracy.

The cellular topological state has a tensor network representation (see Figs. 2 and 4). We can also compute groundstate degeneracy using the trace of the tensor network. Each link of the tensor network has a label $a=1,2,3,4$. The label 1 corresponds to a stripe of $Z_{2}$ topological order in the trivial sector. The label 2,3,4 corresponds to a stripe in the nontrivial sectors. Applying an open $e$-string operator $W_{e}$ connecting the two boundaries to the trivial sector produces the sector 2 (see Fig. 6). Similarly, applying the open $m$-string ( $f$-string) operator $W_{m}\left(W_{f}\right)$ connecting the two boundaries to the trivial sector produces the sector 3 (the sector 4 ).

A ground state of the cellular topological phase is given by the stripes of $Z_{2}$ topological orders, all in the trivial sector (i.e., with label $a=1$ on all links). Now we apply a loop $e$-string operator $W_{e}^{\text {loop }}$ on some links to make them a small loop of sector 2 (see Fig. 8). The configuration corresponds to another degenerate ground state. Thus each hexagon contributes a factor 2 to the ground-state degeneracy.

When the separation between vertices is small, the operators $W_{e}^{\text {loop }}$ are local operators. We may include such operators in the Hamiltonian $\delta H=J \sum W_{e}^{\text {loop }}$. The new Hamiltonian no longer commutes with $m$-string operators $W_{m}$. So $\delta H$ splits the ground-state degeneracy. The new ground states are believed to have a finite degeneracy independent of system size.

The condensation $m_{1} m_{2} m_{3}$ at the $1+1 \mathrm{D}$ topological order $\mathcal{C}_{1}$ implies that we can create three $m$ particles on the neighboring three $2+1 \mathrm{D} Z_{2}$ topological orders, which form a small triangle in the dual honeycomb lattice. Putting many small triangles together gives us a loop in the dual honeycomb
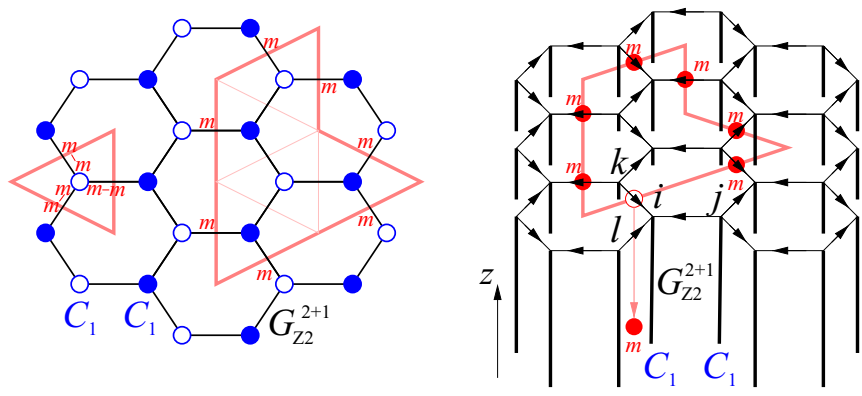

FIG. 9. In the cellular topological state $\left(\mathcal{G}_{Z_{2}}^{2+1}, \mathcal{C}_{1}, \mathcal{C}_{1}\right)$, the $m$ particles must form a closed loop in the dual honeycomb lattice.

lattice formed by the $m$ particles (see Fig. 9). However, the $z$ coordinates of the $m$ particles can be arbitrary.

But if we add the $\delta H=J \sum W_{e}^{\text {loop }}$ term to the Hamiltonian, it will confine two $m$ particles in the same stripe of the $Z_{2}$ topological order. In this case the above loop of the $m$ particles must have similar $z$ coordinates in order to reduce the energy.

Those properties suggest that the cellular topological state $\left(\mathcal{G}_{Z_{2}}^{2+1}, \mathcal{C}_{1}, \mathcal{C}_{1}\right)$ is a $3+1 \mathrm{D} Z_{2}$ topological order $\mathcal{G}_{Z_{2}}^{3+1}$. The freemoving $e$ particle is the pointlike $Z_{2}$ charge in $\mathcal{G}_{Z_{2}}^{3+1}$. The loop of $m$ particles is the $Z_{2}$-flux loop in $\mathcal{G}_{Z_{2}}^{3+1}$.

\section{Cellular topological state $\left(\mathcal{G}_{Z_{2}}^{2+1}, \mathcal{C}_{2}, \mathcal{C}_{2}\right)$}

The cellular topological state $\left(\mathcal{G}_{Z_{2}}^{2+1}, \mathcal{C}_{2}, \mathcal{C}_{2}\right)$ is also a gapped liquid state-a $3+1 \mathrm{D} Z_{2}^{f}$ topological ordered state $\mathcal{G}_{Z_{2}^{f}}^{3+1}$ described by twisted $Z_{2}$ gauge theory where the pointlike $Z_{2}$ charge is a fermion [79].

The $1+1 \mathrm{D}$ topological order $\mathcal{C}_{2}$ has a condensation of $f_{1} f_{2}, f_{2} f_{3}$, and $f_{3} f_{1}$, for the excitations in the connected $2+1 \mathrm{D}$ topological orders. This means that the $f$ particles can freely move between the $2+1 \mathrm{D}$ topological orders $\mathcal{G}_{Z_{2}}^{2+1}$ connected by the $1+1 \mathrm{D}$ topological order $\mathcal{C}_{2}$ (see Fig. 10). In other words, the $f$ particle can move freely in the whole $3 \mathrm{D}$ space, which corresponds to the pointlike $Z_{2}$ charge in the $3+1 \mathrm{D} Z_{2}^{f}$ topological order $\mathcal{G}_{Z_{2}^{f}}^{3+1}$. Similarly, the loop of $m$ particles is the $Z_{2}$-flux loop in $\mathcal{G}_{Z_{2}^{f}}^{3+1}$.

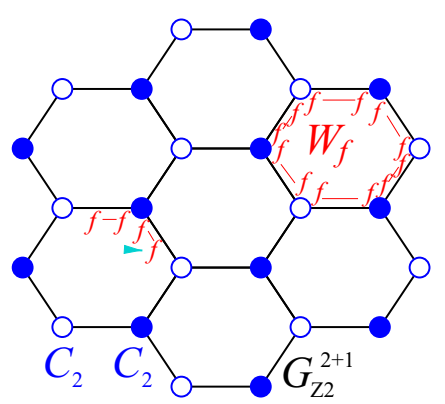

FIG. 10. In the cellular topological state $\left(\mathcal{G}_{Z_{2}}^{2+1}, \mathcal{C}_{2}, \mathcal{C}_{2}\right)$, the $f$ particle can move freely in 3D space. 


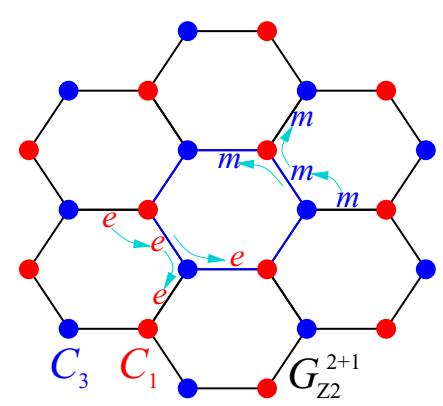

FIG. 11. In the cellular topological state $\left(\mathcal{G}_{Z_{2}}^{2+1}, \mathcal{C}_{1}, \mathcal{C}_{3}\right)$, the $e$ particle can move across the $\mathcal{C}_{1}$ boundary, and the $m$ particle can move across the $\mathcal{C}_{3}$ boundary. However, the long distant motion of $e$ and $m$ particles are blocked.

\section{Cellular topological state $\left(\mathcal{G}_{Z_{2}}^{2+1}, \mathcal{C}_{1}, \mathcal{C}_{3}\right)$}

The cellular topological state $\left(\mathcal{G}_{Z_{2}}^{2+1}, \mathcal{C}_{1}, \mathcal{C}_{3}\right)$ is a gapped nonliquid state, which is a fracton state with fractal excitations. In such a cellular topological state, the $e$ particle $(m$ particle) can move across the $\mathcal{C}_{1}\left(\mathcal{C}_{3}\right)$ boundaries (see Fig. 11). But the motion of the $e$ particle ( $m$ particle) is blocked by the $\mathcal{C}_{3}\left(\mathcal{C}_{1}\right)$ boundaries. To move across the $\mathcal{C}_{3}\left(\mathcal{C}_{1}\right)$ boundaries, the $e$ particle ( $m$ particle) must split into two (see Fig. 11). So the $e$ and $m$ particles cannot move freely in the $x-y$ direction, indicating that the cellular topological state may be a nonliquid state. However, the $e$ particle and $m$ particle can move freely in the $z$ direction within a stripe of $Z_{2}$ topological order (see Fig. 9).

Remember that a ground state of the cellular topological phase is given by the stripes of $Z_{2}$ topological orders, all in the trivial sector (i.e., with label $a=1$ on all links). Now we apply the $m$-string operators $W_{m}$ on some links to make them the sector 3 . The created $m$-particle bound state on a vertex must be able to condense on the boundary. In this case, we create another degenerate ground state (see Fig. 12). We can also apply the $e$-string operators $W_{e}$ on some links to make them the sector 2 . The created $e$-particle bound state on the boundary must be able to condense on the boundary (the resulting configuration is similar to Fig. 12). This way, we obtain another degenerate ground state. We can also apply the $e$-string and $m$-string operators together to obtain new degenerate ground states. Counting all such configurations gives us the ground-state degeneracy. We note that different

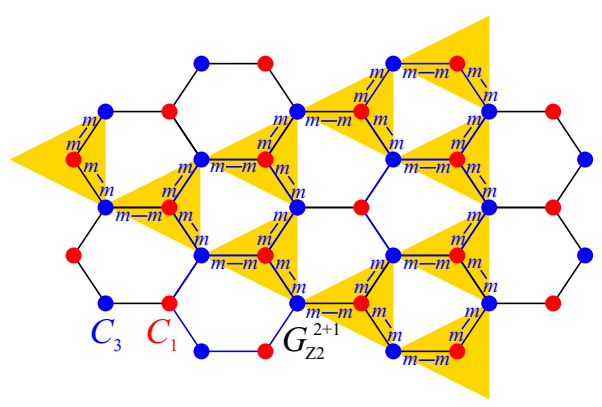

FIG. 12. A configuration in the ground state. The unmarked links are in sector 1 . The links marked by $m-m$ are in sector 3 .

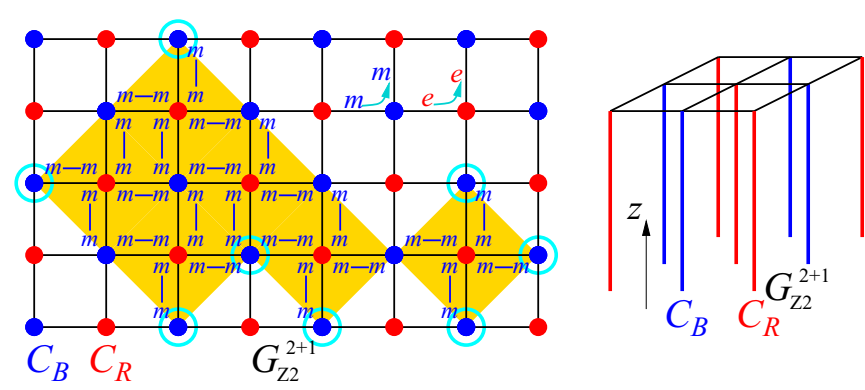

FIG. 13. A configuration of the cellular topological state. The unmarked links are in sector 1 . The links marked by $m-m$ are in sector 3 . Only the vertices in the blue circle cost energy.

degenerate ground states have a large separation of code distance, which increases with system size.

From Fig. 12, we see that, in the ground state, the links in sector 3 form many small triangles. A corner of a triangle must connect to one and only one corner of another triangle. This way, the links in sector 3 form a fractal (see Fig. 12). This implies that the cellular topological state $\left(\mathcal{G}_{Z_{2}}^{2+1}, \mathcal{C}_{1}, \mathcal{C}_{3}\right)$ is a fracton state with fractal excitations.

If a corner of a triangle is not connected to any corner triangle, such a corner will represent a pointlike excitation. But the $x-y$ motion for such a pointlike excitation is highly restricted, like the point excitations in Haah's cubic code. These kinds of point excitations are called fractons. We see fractons are created at the corners of the fractal operator. However, fractons can move freely in the $z$ direction within a stripe of $Z_{2}$ topological order.

We believe that the cellular topological states, $\left(\mathcal{G}_{Z_{2}}^{2+1}, \mathcal{C}_{1}, \mathcal{C}_{2}\right),\left(\mathcal{G}_{Z_{2}}^{2+1}, \mathcal{C}_{1}, \mathcal{C}_{4}\right),\left(\mathcal{G}_{Z_{2}}^{2+1}, \mathcal{C}_{2}, \mathcal{C}_{3}\right),\left(\mathcal{G}_{Z_{2}}^{2+1}, \mathcal{C}_{3}, \mathcal{C}_{4}\right)$. are similar to the cellular topological states $\left(\mathcal{G}_{Z_{2}}^{2+1}, \mathcal{C}_{1}, \mathcal{C}_{3}\right)$ discussed above. They should also be fracton states with fractal excitations.

\section{E. A cellular topological state on square column lattice}

In this section we consider a cellular topological state on a lattice formed by square columns (see Fig. 13). The stripes in the $z$ direction are occupied by $2+1 \mathrm{D} Z_{2}$ topological order. The red and blue vertical lines are two kinds of boundaries, $\mathcal{C}_{R}$ and $\mathcal{C}_{B}$, of those $Z_{2}$ topological orders:

$$
\begin{aligned}
\mathcal{Z}\left(\mathcal{C}_{R}\right) & =\mathcal{G}_{Z_{2}}^{2+1} \otimes \mathcal{G}_{Z_{2}}^{2+1} \otimes \mathcal{G}_{Z_{2}}^{2+1} \otimes \mathcal{G}_{Z_{2}}^{2+1}, \\
\mathcal{Z}\left(\mathcal{C}_{B}\right) & =\mathcal{G}_{Z_{2}}^{2+1} \otimes \mathcal{G}_{Z_{2}}^{2+1} \otimes \mathcal{G}_{Z_{2}}^{2+1} \otimes \mathcal{G}_{Z_{2}}^{2+1} .
\end{aligned}
$$

The two boundaries are characterized by the following condensing topological excitations (the generators):

$$
\begin{aligned}
& \mathcal{C}_{R}: e_{1} e_{2}, e_{1} e_{3}, e_{1} e_{4}, m_{1} m_{2} m_{3} m_{4}, f_{1} f_{2} f_{3} f_{4}, \\
& \mathcal{C}_{B}: m_{1} m_{2}, m_{1} m_{3}, m_{1} m_{4}, e_{1} e_{2} e_{3} e_{4}, f_{1} f_{2} f_{3} f_{4} .
\end{aligned}
$$

From the condensing particles on the boundaries, we see that the $e$ particle can move across the $\mathcal{C}_{R}$ boundaries, while the $m$ particle can move across the $\mathcal{C}_{B}$ boundaries. But the arrangement of the $\mathcal{C}_{R}$ and $\mathcal{C}_{B}$ boundaries is such that the long distant motion of the $e$ and $m$ particles is blocked and they cannot move freely in the $x-y$ direction. This suggests the cellular topological state to be a nonliquid state. 


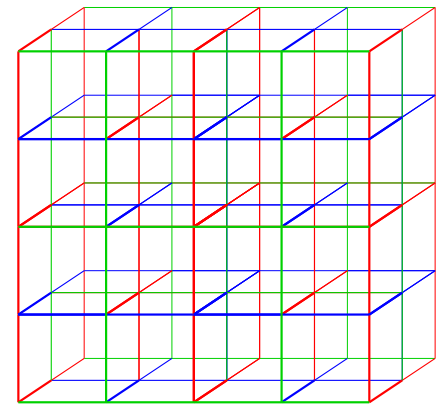

FIG. 14. A cellular topological state on cubic lattice. The square faces are occupied by $2+1 \mathrm{D} Z_{2}$ topological order, and the red, blue, and green lines correspond to three kinds of $1+1 \mathrm{D}$ anomalous topological orders $\mathcal{C}_{R}, \mathcal{C}_{B}$, and $\mathcal{C}_{G}$.

If all the links are in sector 1 , then we have a minimal energy ground state. If we change some links to sector 3 (marked by $m-m$ in Fig. 13), we will get an excited state. We may group the sector-3 links into small diamonds (see Fig. 13). Only the vertices that touch an odd number of the diamonds cost a finite energy (see Fig. 13), and correspond to a fracton. A fracton cannot move by itself in $x-y$ directions. Only a pair of fractons can move in a certain way in $x-y$ directions. But a fracton can move freely and independently in the $z$ direction. The fractons are created at the corner of diamond-shaped membrane operators. Those properties suggest that the constructed cellular topological state is a type-I fracton state.

\section{F. A cellular topological state on cubic lattice}

Now, we consider a cellular topological state on a cubic lattice column (see Fig. 14), which is a generalization of the square column model in the last section. The square faces of the cubic lattice are occupied by $2+1 \mathrm{D} Z_{2}$ topological order. The red, blue, and green lines are three kinds of boundaries, $\mathcal{C}_{R}, \mathcal{C}_{B}$, and $\mathcal{C}_{G}$ of those $Z_{2}$ topological orders:

$$
\begin{aligned}
\mathcal{Z}\left(\mathcal{C}_{R}\right) & =\mathcal{G}_{Z_{2}}^{2+1} \otimes \mathcal{G}_{Z_{2}}^{2+1} \otimes \mathcal{G}_{Z_{2}}^{2+1} \otimes \mathcal{G}_{Z_{2}}^{2+1}, \\
\mathcal{Z}\left(\mathcal{C}_{B}\right) & =\mathcal{G}_{Z_{2}}^{2+1} \otimes \mathcal{G}_{Z_{2}}^{2+1} \otimes \mathcal{G}_{Z_{2}}^{2+1} \otimes \mathcal{G}_{Z_{2}}^{2+1}, \\
\mathcal{Z}\left(\mathcal{C}_{G}\right) & =\mathcal{G}_{Z_{2}}^{2+1} \otimes \mathcal{G}_{Z_{2}}^{2+1} \otimes \mathcal{G}_{Z_{2}}^{2+1} \otimes \mathcal{G}_{Z_{2}}^{2+1} .
\end{aligned}
$$

The above three boundaries are characterized by the following condensing topological excitations (the generators):

$$
\begin{aligned}
& \mathcal{C}_{R}: e_{1} e_{2}, e_{1} e_{3}, e_{1} e_{4}, m_{1} m_{2} m_{3} m_{4}, f_{1} f_{2} f_{3} f_{4}, \\
& \mathcal{C}_{B}: m_{1} m_{2}, m_{1} m_{3}, m_{1} m_{4}, e_{1} e_{2} e_{3} e_{4}, f_{1} f_{2} f_{3} f_{4}, \\
& \mathcal{C}_{G}: f_{1} f_{2}, f_{1} f_{3}, f_{1} f_{4}, e_{1} e_{2} e_{3} e_{4}, m_{1} m_{2} m_{3} m_{4} .
\end{aligned}
$$

From the condensing particles on the boundaries, we see that the $e$ particle can move across the $\mathcal{C}_{R}$ boundaries, the $m$ particle can move across the $\mathcal{C}_{B}$ boundaries, and the $f$ particle can move across the $\mathcal{C}_{G}$ boundaries. But the arrangement of the $\mathcal{C}_{R}, \mathcal{C}_{B}$, and $\mathcal{C}_{G}$ boundaries is such that the long distant motion of the $e, m$, and $f$ particles is blocked and they cannot move freely in any direction. In other words, they are localized in a finite region. To move further, those particles must split into more and more particles. This suggests the cellular topological state in Fig. 14 to be a nonliquid state.
In particular, the structure described in Fig. 13 also appears in the cubic cellular model, and gives rise to pointlike excitations with constrained motion.

\section{REVERSE RENORMALIZATION AND GENERIC CONSTRUCTION}

To systematically understand and to classify a gapped liquid state (such as a topologically ordered state), we perform wave-function renormalization by removing the unentangled degrees of freedom [77,80-82]. We hope to obtain a fixedpoint wave function which gives us a classifying understanding of the gapped liquid states. We also hope the fixed-point wave function is described by a topological quantum field theory which is not dependent on the lattice details.

However, for gapped nonliquid states, due to their intrinsic foliation or cellular structure, the above general approach does not work. In particular, we should not expect to have a quantum field theory to describe a nonliquid state. (But a quantum field theory with an explicit layer structure may work [53].) On the other hand, we still hope to obtain some kind of fixed-point wave functions for nonliquid states, so that we can have a systematic and classifying understanding of nonliquid states.

Here we like to propose a reverse renormalization approach to obtain the fixed-point wave functions for nonliquid states. In such an approach, we add unentangled degrees of freedom to our systems to separate the layers in the foliation or cellular structure. After many steps of renormalization, we get $3+1 \mathrm{D}$ gapped liquid states between layers (i.e., within a cell), such as topologically ordered states or SET/SPT states if we have symmetry. (In our previous discussions, we have assumed the $3+1 \mathrm{D}$ gapped liquid states to be trivial product states.) On the layers, we have $2+1 \mathrm{D}$ anomalous topological orders, which are the domain walls separating the neighboring $3+1 \mathrm{D}$ topological orders. The layers join at edges, which correspond to $1+1 \mathrm{D}$ anomalous topological orders. The edges join at vertices, which correspond to $0+1 \mathrm{D}$ anomalous topological orders (see Fig. 1).

The above reverse renormalization understanding of nonliquid states suggests the following general construction. We first decompose the 3D space into cells (see Fig. 1). We assign (possibly different) $3+1 \mathrm{D}$ topological orders to the $3 \mathrm{D}$ cells, assign $2+1 \mathrm{D}$ anomalous topological orders to the $2 \mathrm{D}$ surfaces, assign $1+1 \mathrm{D}$ anomalous topological orders to the $1 \mathrm{D}$ edges, and assign $0+1 \mathrm{D}$ anomalous topological orders to the $0 \mathrm{D}$ vertices. (Without symmetry, the $0+1 \mathrm{D}$ anomalous topological orders are always trivial [42].) This is a quite general construction, which may cover all the nonliquid states. However, some constructions may give rise to ground-state degeneracies that can be lifted by local operators. We need to include those local operators to lift the degeneracies and to stabilize the constructed states. Also, different constructions may lead to the same gapped nonliquid phase. Finding the equivalence relations between different constructions is an every important issue.

Our construction also works if there is on-site symmetry, by requiring the (anomalous) topological orders in various dimensions to have the same symmetry. In the presence of 
space group symmetry, we need to choose the cellular structure to have the space group symmetry. We also need to choose (anomalous) topological orders in various dimensions to have the proper symmetries, as discussed in Refs. [45-48].

After posting this paper, the author became aware of a prior unpublished work (now posted as Ref. [83]) where a very similar construction, based on the defect network in a $3+1 \mathrm{D}$ topological quantum field theory, was proposed. The defect planes and defect lines correspond to the (anomalous) $2+1 \mathrm{D}$ and $1+1 \mathrm{D}$ topological orders in this paper. Later, another similar construction was proposed in Ref. [84].

\section{ACKNOWLEDGMENTS}

I would like to thank Xie Chen and Kevin Slagle for bringing the above work to my attention. This work is motivated by the presentations in the Annual Meeting of Simons Collaboration on Ultra-Quantum Matter, where the issue of the fixed point field theory for fracton phases was discussed. This research was partially supported by NSF Grant No. DMS-1664412. This work was also partially supported by the Simons Collaboration on Ultra-Quantum Matter, which is a grant from the Simons Foundation (Grant No. 651440).
[1] L. D. Landau, Phys. Z. Sowjetunion 11, 26 (1937).

[2] L. D. Landau, Phys. Z. Sowjetunion 11, 545 (1937).

[3] X. Chen, Z.-C. Gu, and X.-G. Wen, Phys. Rev. B 82, 155138 (2010).

[4] B. Zeng and X.-G. Wen, Phys. Rev. B 91, 125121 (2015).

[5] B. Swingle and J. McGreevy, Phys. Rev. B 93, 045127 (2016).

[6] X. G. Wen, Phys. Rev. B 40, 7387 (1989).

[7] X. G. Wen, Int. J. Mod. Phys. B 04, 239 (1990).

[8] E. Keski-Vakkuri and X.-G. Wen, Int. J. Mod. Phys. B 07, 4227 (1993).

[9] X.-G. Wen, Phys. Rev. B 65, 165113 (2002).

[10] A. M. Essin and M. Hermele, Phys. Rev. B 87, 104406 (2013).

[11] L.-Y. Hung and X.-G. Wen, Phys. Rev. B 87, 165107 (2013).

[12] C. Xu, Phys. Rev. B 88, 205137 (2013).

[13] A. Mesaros and Y. Ran, Phys. Rev. B 87, 155115 (2013).

[14] X. Chen, F. J. Burnell, A. Vishwanath, and L. Fidkowski, Phys. Rev. X 5, 041013 (2015).

[15] L. Chang, M. Cheng, S. X. Cui, Y. Hu, W. Jin, R. Movassagh, P. Naaijkens, Z. Wang, and A. Young, J. Phys. A: Math. Theor. 48, 12FT01 (2015).

[16] M. Cheng, Z.-C. Gu, S. Jiang, and Y. Qi, Phys. Rev. B 96, 115107 (2017).

[17] C. Heinrich, F. Burnell, L. Fidkowski, and M. Levin, Phys. Rev. B 94, 235136 (2016).

[18] Z.-C. Gu and X.-G. Wen, Phys. Rev. B 80, 155131 (2009).

[19] X. Chen, Z.-X. Liu, and X.-G. Wen, Phys. Rev. B 84, 235141 (2011).

[20] X. Chen, Z.-C. Gu, Z.-X. Liu, and X.-G. Wen, Phys. Rev. B 87, 155114 (2013).

[21] C. Chamon, Phys. Rev. Lett. 94, 040402 (2005).

[22] J. Haah, Phys. Rev. A 83, 042330 (2011).

[23] W. Shirley, K. Slagle, Z. Wang, and X. Chen, Phys. Rev. X 8, 031051 (2018).

[24] W. Shirley, K. Slagle, and X. Chen, SciPost Phys. 6, 015 (2019).

[25] S. Vijay, J. Haah, and L. Fu, Phys. Rev. B 94, 235157 (2016).

[26] X. Chen, Z.-C. Gu, and X.-G. Wen, Phys. Rev. B 83, 035107 (2011).

[27] N. Schuch, D. Pérez-García, and I. Cirac, Phys. Rev. B 84, 165139 (2011).

[28] M. Barkeshli, P. Bonderson, M. Cheng, and Z. Wang, Phys. Rev. B 100, 115147 (2019).

[29] T. Lan, L. Kong, and X.-G. Wen, Phys. Rev. B 95, 235140 (2017).
[30] T. Lan, L. Kong, and X.-G. Wen, Commun. Math. Phys. 351, 709 (2016).

[31] T. Lan, L. Kong, and X.-G. Wen, Phys. Rev. X 8, 021074 (2018).

[32] T. Lan and X.-G. Wen, Phys. Rev. X 9, 021005 (2019).

[33] C. Zhu, T. Lan, and X.-G. Wen, Phys. Rev. B 100, 045105 (2019).

[34] A. Kapustin, arXiv:1404.6659.

[35] Z.-C. Gu and X.-G. Wen, Phys. Rev. B 90, 115141 (2014).

[36] A. Kapustin, R. Thorngren, A. Turzillo, and Z. Wang, J. High Energ. Phys. 12 (2015) 052.

[37] D. Gaiotto and A. Kapustin, Int. J. Mod. Phys. A 31, 1645044 (2016).

[38] D. S. Freed and M. J. Hopkins, arXiv:1604.06527.

[39] A. Kapustin and R. Thorngren, J. High Energ. Phys. 10 (2017) 080.

[40] Q.-R. Wang and Z.-C. Gu, Phys. Rev. X 8, 011055 (2018).

[41] M. Pretko, X. Chen, and Y. You, Int. J. Mod. Phys. A 35, 2030003 (2020).

[42] L. Kong and X.-G. Wen, arXiv:1405.5858.

[43] X.-G. Wen, Phys. Rev. D 88, 045013 (2013).

[44] A. Kapustin, arXiv:1403.1467.

[45] X. Chen, Y.-M. Lu, and A. Vishwanath, Nat. Commun. 5, 3507 (2014).

[46] H. Song, S.-J. Huang, L. Fu, and M. Hermele, Phys. Rev. X 7, 011020 (2017).

[47] S.-J. Huang, H. Song, Y.-P. Huang, and M. Hermele, Phys. Rev. B 96, 205106 (2017).

[48] Z. Song, C. Fang, and Y. Qi, Nat. Commun. 11, 4197 (2020).

[49] H. Ma, E. Lake, X. Chen, and M. Hermele, Phys. Rev. B 95, 245126 (2017).

[50] K. Slagle and Y. B. Kim, Phys. Rev. B 96, 165106 (2017).

[51] S. Vijay, arXiv:1701.00762.

[52] A. Prem, S.-J. Huang, H. Song, and M. Hermele, Phys. Rev. X 9, 021010 (2019).

[53] K. Slagle, D. Aasen, and D. Williamson, SciPost Phys. 6, 043 (2019).

[54] Y. Fuji, Phys. Rev. B 100, 235115 (2019).

[55] C. Xu, arXiv:cond-mat/0602443.

[56] Z.-C. Gu and X.-G. Wen, arXiv:gr-qc/0606100 (2006).

[57] Z.-C. Gu and X.-G. Wen, Nucl. Phys. B 863, 90 (2012).

[58] T. Lan and X.-G. Wen, Phys. Rev. B 90, 115119 (2014). 
[59] T. Lan, J. C. Wang, and X.-G. Wen, Phys. Rev. Lett. 114, 076402 (2015).

[60] Y. Hu, Y. Wan, and Y.-S. Wu, Chin. Phys. Lett. 34, 077103 (2017).

[61] T. Lan, X. Wen, L. Kong, and X.-G. Wen, Phys. Rev. Research 2, 023331 (2020).

[62] A. Kapustin and N. Saulina, Nucl. Phys. B 845, 393 (2011).

[63] A. Kitaev and L. Kong, Commun. Math. Phys. 313, 351 (2012).

[64] J. C. Wang and X.-G. Wen, Phys. Rev. B 91, 125124 (2015).

[65] L. Kong, Nucl. Phys. B 886, 436 (2014).

[66] L.-Y. Hung and Y. Wan, Int. J. Mod. Phys. B 28, 1450172 (2014).

[67] L.-Y. Hung and Y. Wan, Phys. Rev. Lett. 114, 076401 (2015).

[68] M. Mignard and P. Schauenburg, arXiv:1708.02796.

[69] P. Bonderson, C. Delaney, C. Galindo, E. C. Rowell, A. Tran, and Z. Wang, arXiv:1805.05736.

[70] X. Wen and X.-G. Wen, arXiv:1908.10381.
[71] E. Rowell, R. Stong, and Z. Wang, Commun. Math. Phys. 292, 343 (2009)

[72] X.-G. Wen, Nat. Sci. Rev. 3, 68 (2015).

[73] L. Kong, X.-G. Wen, and H. Zheng, arXiv:1502.01690.

[74] L. Kong, X.-G. Wen, and H. Zheng, Nucl. Phys. B 922, 62 (2017).

[75] N. Read and S. Sachdev, Phys. Rev. Lett. 66, 1773 (1991).

[76] X. G. Wen, Phys. Rev. B 44, 2664 (1991).

[77] F. Verstraete and J. I. Cirac, arXiv:cond-mat/0407066.

[78] M. Levin and C. P. Nave, Phys. Rev. Lett. 99, 120601 (2007).

[79] M. Levin and X.-G. Wen, Phys. Rev. B 67, 245316 (2003).

[80] G. Vidal, Phys. Rev. Lett. 99, 220405 (2007).

[81] M. Aguado and G. Vidal, Phys. Rev. Lett. 100, 070404 (2008).

[82] Z.-C. Gu, M. Levin, and X.-G. Wen, Phys. Rev. B 78, 205116 (2008).

[83] D. Aasen, B. Bulmash, A. Prem, K. Slagle, and D. J. Williamson, arXiv:2002.05166.

[84] J. Wang, arXiv:2002.12932. 